\title{
AUTOMATIC FLAMINGO DETECTION USING A MULTIPLE BIRTH AND DEATH PROCESS
}

\author{
Stig Descamps ${ }^{1}$, Xavier Descombes ${ }^{1}$, Arnaud Béchet ${ }^{2}$, Josiane Zerubia ${ }^{1}$ \\ ${ }^{1}$ Equipe-Projet Ariana, INRIA/I3S \\ 2004 route des Lucioles, BP 93, 06902 Sophia Antipolis Cedex (France) \\ E-mail : Firstname.Lastname@inria.fr \\ ${ }^{2}$ Tour du Valat \\ Le Sambuc, 13200 Arles (France)
}

\begin{abstract}
Here we present a new approach to automatically detect and count breeding Greater Flamingos (Phoenicopterus Roseus) on aerial photographs of their colonies. We consider a stochastic approach based on object processes also called marked point processes. The objects represent flamingos which are defined as ellipses. We formulate a Gibbs density, associated with the marked point process of ellipses, which is defined w.r.t a Poisson measure. Thus, the issue is reduced to an energy minimization, where the energy is composed of a regularization term (prior density), which introduces some constraints on the objects and their interactions, and a data term, which links the objects to the features to be extracted in the image. Then, we sample the process to extract the configuration of objects minimizing the energy by a new and fast birth-and-death dynamics, leading to the total number of birds. This approach gives counts with good precision compared to manual counts. Additionally, this approach does not need image pre-processing or supervision of the extraction by an operator thus considerably reducing the overall processing time required to get the estimate.
\end{abstract}

Index Terms - bird colony, Object extraction, marked point processes, stochastic modeling, birth/death dynamics.

\section{INTRODUCTION}

During breeding seasons, flamingos gather in a large colony. Thus, specialists take advantage of it to asset the number of flamingos in the colony. Since the 60's, several techniques have been developed to estimate the number of flamingos from aerial images. Most of them are highly supervised. They are based on an expert counting on some predefined small areas. The total number of flamingos is then estimated from an interpolation procedure. This methodology is therefore time consuming for the experts and lacks precision. General sofware for object detection based on classical image processing tools such as mathematical morphology [1] or template matching [2] appear to be unsatisfactory. A more dedicated approach embedding a geometric model of the flamingos and some constraints on their spatial repartition is required.

We propose in this paper, a new method for automatically estimating the size of flamingo populations based on object processes. We consider an ellipse as our reference object to model flamingos. Indeed, on aerial images, flamingos look like ellipses. Greater flamingos are mainly covered with white plumage. This fact gives a feature to evaluate, with the Bhattacharrya distance, the contrast between the background and flamingos themselves. The density associated with the marked point process of ellipses is defined with respect to the Poisson measure. Moreover, by formulating the model as a Gibbs density, we reduce the problem to an energy minimization. This energy is decomposed into a data term to locate flamingo on the image, and a prior term which introduces constraints between the objects of the configuration.

\section{A MARKED POINT PROCESS MODEL}

\subsection{Definition and notation}

We model aerial images as composed of flamingos whose positions and attributes are some realization of a marked point process $X$, see [3] for more details. $X$ is also a random variable whose realizations are random configurations of objects belonging to a set space $\chi=\mathcal{P} \times \mathcal{M}$, where $\mathcal{P}$ is the position space, and $\mathcal{M}$ the space of the marks. We note $\Phi$ the space of all configurations of a finite number of objects. The probability distribution $\mathcal{P}_{X}($.$) of the stochastic process is uniformly$ continuous with respect to the Poisson measure $\mu($.$) of inten-$ sity $\lambda($.$) on \chi$. Then, by using the Gibbs energy formulation of the process density, we define an energy $U(\mathrm{x})$ as :

$$
\mathcal{P}_{X}(d \mathrm{x})=\frac{1}{Z} \exp (-U(\mathrm{x})) \mu(d \mathrm{x})
$$

where $\mathrm{Z}$ is a normalizing constant. This energy will be minimized on $\Phi$ by the flamingo extraction. It takes into account 
the interactions between the geometric objects (the prior energy $U_{p}(\mathrm{x})$ ), and the way they fit to the data (the data energy $\left.U_{d}(\mathrm{x})\right)$ :

$$
U(\mathrm{x})=U_{p}(\mathrm{x})+U_{d}(\mathrm{x})
$$

\subsection{Objects of interest}

The 2D model, used to extract flamingos, consists of a marked point process of ellipses. The associated set space $\chi$ is :

$\chi=\mathcal{P} \times \mathcal{M}=\left[0, X_{M}\right] \times\left[0, Y_{M}\right] \times\left[a_{m}, a_{M}\right] \times\left[b_{m}, b_{M}\right] \times[0, \pi[$ where $X_{M}$ and $Y_{M}$ are respectively the width and the length of the image I, $\left(a_{m}, a_{M}\right)$ and $\left(b_{m}, b_{M}\right)$ respectively the minimum and the maximum semimajor axis and semiminor axis, and $\theta \in[0, \pi[$ the orientation of the objects.

\subsection{Prior energy}

As we aim at detecting individuals in dense populations, we model flamingos as possibly slightly overlapping ellipses $x_{i} \sim_{r}$ $x_{j}$. Then, the prior energy $U_{p}(\mathrm{x})$ that introduces interactions, penalizes configurations according to the overlapping objects area, see [4] for more details:

$$
U_{p}(\mathrm{x})=\gamma_{p} \sum_{x_{i} \in \mathbf{x}} \max _{x_{j} \sim_{r} x_{i}} \mathcal{A}\left(x_{i}, x_{j}\right)
$$

where $\mathcal{A}\left(x_{i}, x_{j}\right) \in[0,1]$ is an overlapping coefficient and $\gamma_{p}$ is a weight which ponders the repulsion between the objects of the process. Each object is penalized depending on the maximal overlapping it exhibits with neighboring ellipses.

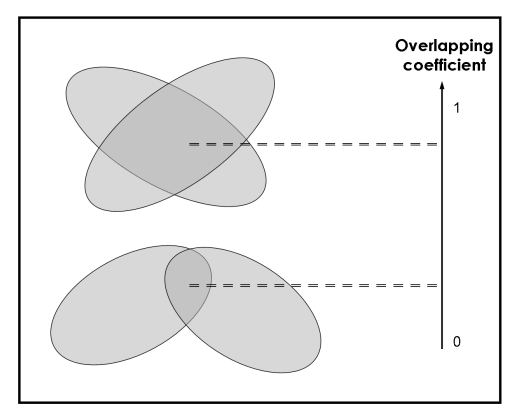

Fig. 1. Overlapping ellipses

\subsection{Data energy}

In flamingo populations, each flamingo can be modeled as a bright ellipse surrounded by a darker background. Thus, we define the boundary of an ellipse $\mathcal{F}(x)$ as the set of $\mathcal{P}$ contained between the given ellipse $x=(p, k)$, where $k=$ $(a, b, \theta)$ are the marks, and a concentric one $x^{\prime}=\left(p, k^{\prime}\right)$, with $k^{\prime}=(a+\rho, b+\rho, \theta)$. This boundary will stand for the background. To evaluate the contrast between the ellipses and the background, we calculate the Bhattacharya distance $d_{B}(x, \mathcal{F}(x))$ between the reflectance distributions of the object and its boundary as follows:

$$
d_{B}(x, \mathcal{F}(x))=1-\int \sqrt{h_{1}(x) h_{2}(x)} d x,
$$

where $h_{1}(x)$ (resp. $h_{2}(x)$ ) is the empirical distribution of the pixels belonging to $x$ (resp. $\mathcal{F}(x)$ ).

The data energy $U_{d}(x)$ associated with the object $x$ is then given by:

$$
U_{d}(x)=\mathcal{Q}_{d}\left(d_{B}(x, \mathcal{F}(x))\right)
$$

where $\mathcal{Q}_{d}\left(d_{B}\right) \in[-1,1]$ is a quality function which gives some positive value to small Bhattacharya distance (weakly contrasted objects) and negative value (well located objects) otherwise.

$$
\mathcal{Q}_{d}\left(d_{B}\right)= \begin{cases}\left(1-\frac{d_{B}}{d_{0}}\right) & \text { si } d_{B}<d_{0} \\ \exp \left(-\frac{d_{B}-d_{0}}{D}\right)-1 & \text { si } d_{B} \geqslant d_{0}\end{cases}
$$

where $D$ is a scale parameter calibrated to 100 .

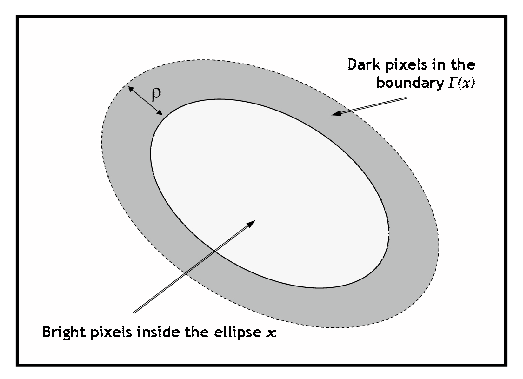

Fig. 2. An ellipse and its boundary

\subsection{Parameter estimation}

The data term involves a parameter $d_{0}$ which can be interprated as the mean value of the contrast between a flamingo and its neighborhood. We propose to estimate it by considering a weighted histogram.

For each pixel $s$ of the image $I$, we compute the data energy $U_{d}^{s}(c)$ corresponding to a disc whose radius is equal to the minimal size of flamingos to be extracted in the image. This map will be used for estimating the value of $d_{0}$. It requires an initial value which has been calibrated to $d_{0}=10$.

We derive a predetection map by computing the following rate:

$$
\forall s \in I, b(s)=1+9 \frac{\max _{t \in I} U_{d}^{t}(c)-U_{d}^{s}(c)}{\max _{t \in I} U_{d}^{t}(c)-\min _{t \in I} U_{d}^{t}(c)}
$$

The higher $b(s)$, the more likely a flamingo is in $s$. 
Usually, flamingo color is not homogeneous within a given image because of the focal of the camera. Thus, we present a local parameter estimation method. To compute $d_{0}$, we need to estimate the mean of flamingo color in small squared area of the initial image. This estimation is decomposed in three steps: the construction of a weighted histogram of the initial image, a first estimation of the parameters and a final filtering of the parameter previously estimated over the image:

- The weighted histogram is simply obtained by constructing the histogram of the initial image whose pixels are weighted by the respective pixels of the predetection map. - For each

squared region, we extract the mean of the colour in the considered region by detecting the maximum of the weighted histogram. - Once done for every squared region of the image,

we compute a spatial filtering of the previous parameters to correct biased estimates in low density areas.

$d_{0}$ is then computed from this flamingo mean radiometry estimation.

\section{BIRTH AND DEATH DYNAMICS}

For optimizing the model, we consider a simulated annealing based on a birth and death process. This process has first been proposed in [5], where the proof of the convergence is given in the case of disks, the generalization to ellipses being straightforward. lows:

The algorithm simulating the process is defined as fol-

- Main program: initialize the inverse temperature parameter $\beta=\beta_{0}=50$ and the discretization step $\delta=$ $\delta_{0}=20000$ and alternate birth and death steps

- Birth step: for each $s \in S$, if no object is already alive, we add an object in $s$ with probability $\delta B(s)$ where $B(s)$ is derived from the predetection map:

$$
\forall s \in I, B(s)=\frac{z b(s)}{\sum_{t \in I} b(t)}
$$

where $z$ is a parameter of the process.

- Sorting step: once the birth step is finished, we compute the data term $U_{d}^{s}(c)$ of the current configuration objects $u_{c}$. Then, we sort them, from increasing, according to their data energy.

- Death step: for each object taken in this order, we compute the death rate as follows:

$$
d\left(u_{c}\right)=\frac{\delta a_{\varphi}\left(u_{c}\right)}{1+\delta a_{\varphi}\left(u_{c}\right)}
$$

where $a_{\varphi}\left(u_{c}\right)=\exp \left(-\varphi U\left(u_{c}\right)\right)$, then the object $u_{c}$ dies with probability $d\left(u_{c}\right)$.
- Convergence test: if the process has not converged, decrease the temperature and the discretization step by a given factor and go back to the birth step. The convergence is obtained when all the objects added during the birth step, and only these ones, have been killed during the death step.

\section{RESULTS AND DISCUSSIONS}

We present in figure 4 and figure 3 two results on real aerial images. Computation took a couple of minutes for each result, which depends on both the number of objects to be extracted and also on the size of the image.
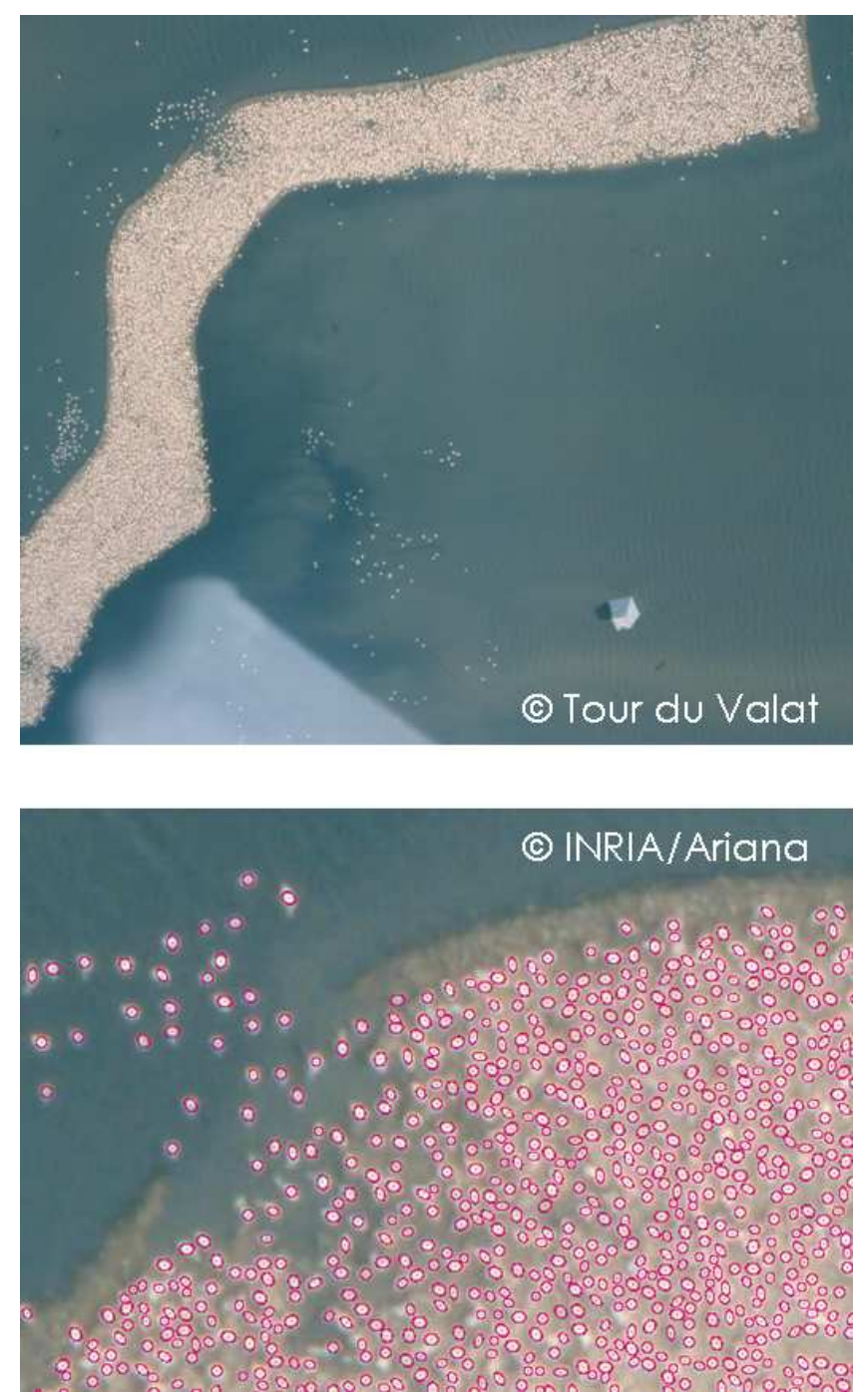

Fig. 3. Top: initial population image. Bottom: Sample of the extraction

To evaluate these results, we have computed the detection rate compared to a manual extraction, executed by an expert.The different datasets have been divided in three classes 


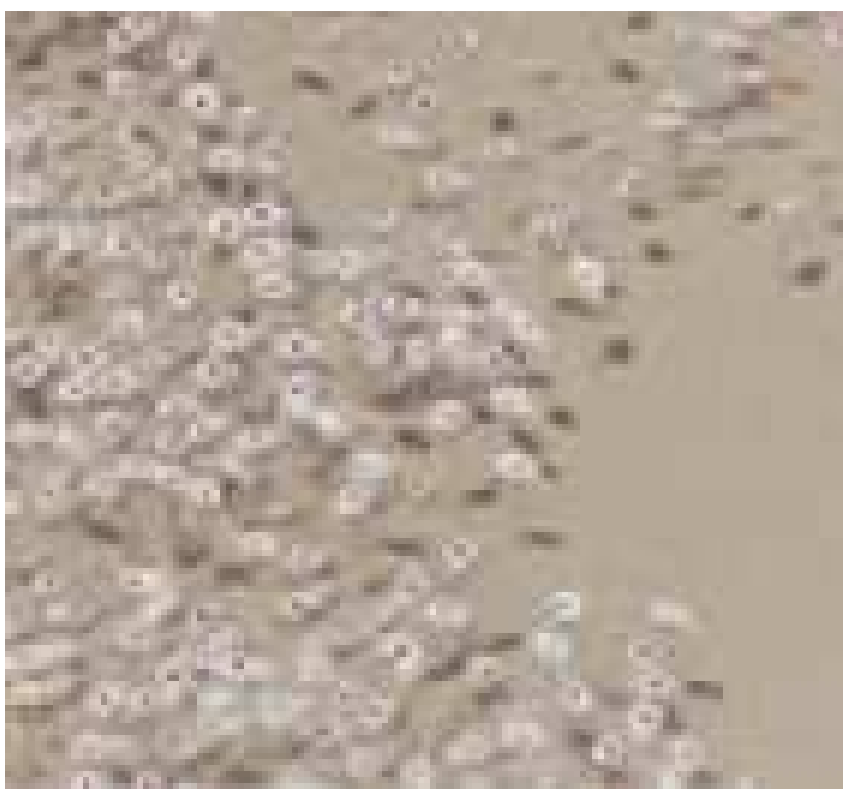

Fig. 4. Sample of the extraction on a Mauritanian colony (only the ellipse center is pointed)

depending on the image quality and the population density. The false alarm rate is always negligible and the detection rate is given in figure 5. In most cases, the desagreement between the expert and our detection corresponds to ambiguous cases where no decision can reasonably be taken from the image alone.

\section{CONCLUSION}

In this paper, we proposed an algorithm to automatically extract flamingo populations from aerial images. Based on a stochastic geometry

approach, we have shown the efficiency of the detection on low resolution images where it is even tricky for the human eye to distinguish flamingos between themselves. The automatic paramater estimation gives a major advantage over other current techniques of detection because no preprocessing is needed. Futhermore, execution time requirements are reasonable to obtain flamingo population extractions because of the proposed birth and death process which outperform classical RJMCMC schemes [4].

\section{ACKNOWLEDGEMENTS}

The authors would like to thank Antoine Arnaud from the Tour du Valat for his help in validating the results by an expert. This work has been partially supported by the COLOR 'Flamant' funded by INRIA Sophia Antipolis.

\begin{tabular}{|l|c|c|}
\hline Image/Statistics & Counting difficulty & Good detection rate \\
\hline Tuz06 & & \\
\hline Sample1 & easy & $99 \%$ \\
\hline Sample2 & medium & $99 \%$ \\
\hline Sample3 & medium & $99 \%$ \\
\hline Tuz04 & & \\
\hline Sample1 & easy & $99 \%$ \\
\hline Sample2 & easy & $100 \%$ \\
\hline Sample3 & easy & $100 \%$ \\
\hline Kiaone05 & & \\
\hline Sample1 & hard & $89 \%$ \\
\hline Sample2 & hard & $96 \%$ \\
\hline Sample3 & hard & $91 \%$ \\
\hline Fang02 & & \\
\hline Sample1 & hard & $86 \%$ \\
\hline Sample2 & medium & $93 \%$ \\
\hline Sample3 & easy & $98 \%$ \\
\hline Fang05 & & $92 \%$ \\
\hline Sample1 & medium & $94 \%$ \\
\hline Sample2 & medium & $92 \%$ \\
\hline Sample3 & medium & \\
\hline
\end{tabular}

Fig. 5. Resulting statistics

\section{REFERENCES}

[1] P. Soille, Morphological Image Analysis, Springer Verlag, 1999.

[2] R. Pollock, "Individual Tree Recognition based on Synthetic Tree Crowns Image Model," in Proc. of the International Forum on Automated Interpretation of High Spatial Resolution Digital Imagery for For estry, D.A. Hill and D.G. Leckie, Eds., February 1998, pp. 25-34.

[3] M.N.M. Van Lieshout, "Markov point processes and their applications," Imperial College Press, London, 2000.

[4] G. Perrin, X. Descombes, and J. Zerubia, " $2 \mathrm{~d}$ and 3d vegetation resource parameters assessment using marked point processes," in Proc. International Conference on Pattern Recognition (ICPR), Hong-Kong, août 2006.

[5] X. Descombes, R. Minlos, and E. Zhizhina, "Object extraction using a stochastic birth-and-death dynamics in continuum," in Research Report No 6135, 2007. 